This item was submitted to Loughborough's Research Repository by the author.

Items in Figshare are protected by copyright, with all rights reserved, unless otherwise indicated.

\title{
Conduction mechanisms in anisotropic conducting adhesive assembly
}

PLEASE CITE THE PUBLISHED VERSION

PUBLISHER

(C) Institute of Electrical and Electronics Engineers (IEEE)

LICENCE

CC BY-NC-ND 4.0

\section{REPOSITORY RECORD}

Oguibe, Chucks N., Samjid H. Mannan, David C. Whalley, and David J. Williams. 2019. "Conduction Mechanisms in Anisotropic Conducting Adhesive Assembly". figshare. https://hdl.handle.net/2134/3786. 
This item was submitted to Loughborough's Institutional Repository (https://dspace.lboro.ac.uk/) by the author and is made available under the following Creative Commons Licence conditions.

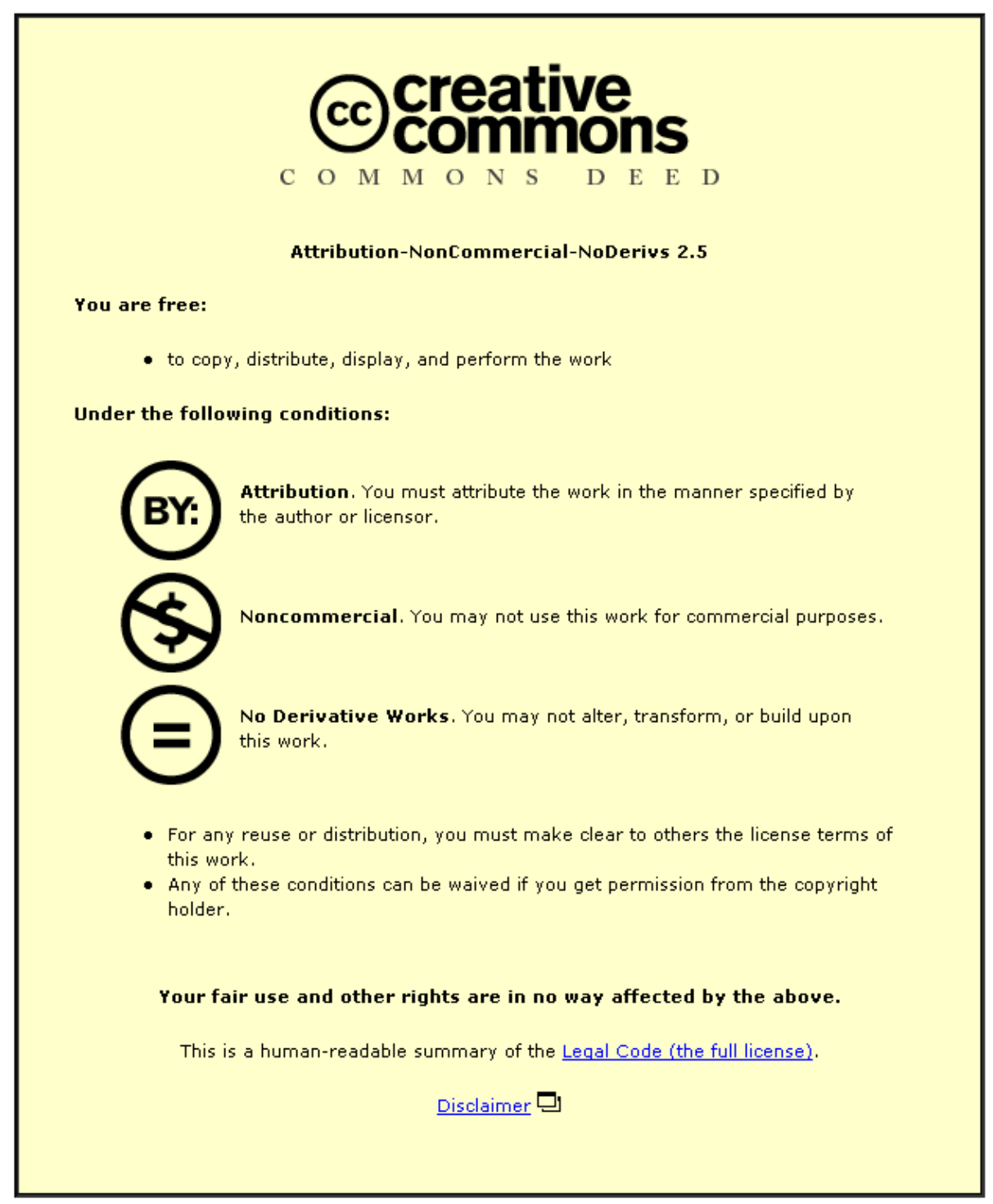

For the full text of this licence, please go to: http://creativecommons.org/licenses/by-nc-nd/2.5/ 


\title{
Conduction Mechanisms in Anisotropic Conducting Adhesive Assembly
}

\author{
Chuks N. Oguibe, Samjid H. Mannan, David C. Whalley, and David J. Williams
}

\begin{abstract}
This paper explores both experimentally and through analytical and computational models, the mechanisms of conduction in flip-chip interconnections made using anisotropic conducting adhesives. A large number of assemblies have been constructed with geometries in the range of $200-500 \mu \mathrm{m}$, and wide variations in their joint resistance were observed to occur both within the same assembly and between assemblies under the same experimental conditions. In order to attempt to explain the origin of these unsatisfactory connections, a series of experiments to measure the linearity of the contact resistance of both high and low resistance joints was made. The results from these measurements show that the large number of low resistance joints are ohmic, while most of the joints of relatively high resistance show resistive heating.

In addition to the linearity measurements, computational models of metallic conduction in solid and polymer cored particles have been constructed to help understand the mechanism of conduction. These models, which are based on the finite element (FE) method, represent typical conductor particles trapped between appropriate substrate and component metallization. The results from the models show that the contact area required to explain the high resistances is small and that the likelihood of obtaining a high resistance through such a small area of metal-to-metal contact is small, thus, giving a strong indication of the presence of high resistivity films at the contact surfaces of the joints.
\end{abstract}

Index Terms - ACA, adhesive, conduction mechanisms, conductive fillers, electric contact, finite element method, ohmic behavior, resistance linearity measurements.

\section{INTRODUCTION}

$\mathbf{T}$ HE increasing demand for fine pitch interconnections capable of reliable operation at temperatures comparable and above those of tin/lead solder joints, and the environmental concerns over the continual use of lead in electronic products, have given rise to new electronic assembly methods. One new and innovative connection technology geared toward achieving increased functionality at a lower total system cost, is anisotropic conducting adhesive (ACA) interconnection. Anisotropic conductive adhesives, when used for flip chip assembly, provide electrical as well as mechanical interconnections. They generally consist of an adhesive polymer

Manuscript received October 1, 1997; revised March 1, 1998. This work was supported by the Engineering and Physical Sciences Research Council (EPSRC) and consortium of industrial partners: Alpha Metals Advanced Products, Eltek Semiconductors Ltd., Lucas Aerospace, Rolls-Royce Plc, IBM (U.K.) Ltd., and Mitel Telecom. This paper was presented at the First IEEE International Symposium on Polymeric Electronics Packaging, Norrkoping, Sweden, October 26-30, 1997. This paper was recommended for publication by Editors J. Liu and J. E. Morris upon evaluation of the reviewers' comments.

The authors are with the Department of Manufacturing Engineering, Loughborough University, Loughborough, Leic. LE11 3TU, U.K.

Publisher Item Identifier S 1070-9886(98)05009-4.

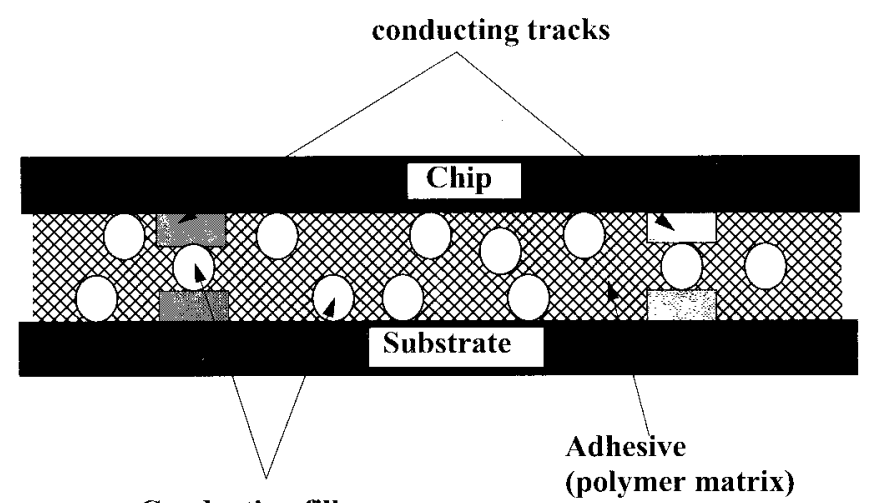

Conducting fillers

(metallic or metal-coated polymer balls)

Fig. 1. Schematic representation of a cross section of an ACA assembly showing the adhesive and conducting fillers.

matrix and fine conductive fillers of metallic, or metal-coated polymer, particles. Electrical conduction is provided by these conductive fillers when trapped between the interconnection areas in the z-axis of the adhesives, i.e., perpendicular to the plane of the board, while electrical isolation is provided in the plane of the adhesive layer. Fig. 1 shows a typical cross section of an ACA assembly, while a review of the application of these materials is given in [1].

In spite of the increasing role of ACA's in the assembly of electronic products, there are concerns about their low conductivity and low yield in some applications. One of the key performance requirements of ACA's is uniformly high conductivity; the requirement for high yield being a good electrical contact under uncontrolled environmental conditions. An accurate prediction of this performance has become increasingly important so as to meet stringent specifications, and to ensure reliability of operation. The challenge, therefore, is to achieve a low joint resistance which is stable over time and environmental stress. In doing this, it is important that the mechanism of electrical conduction at the conducting joints is well understood. This, in turn, will enable the electrical performance under various operating conditions to be predicted accurately.

This paper presents the results of a program of experimental and computational work aimed at understanding the mechanisms of electrical conduction at the conducting joints of interconnections made with anisotropic conducting adhesives. Experiments to investigate the linearity of the contact resistance of flip chip assemblies constructed with geometries in the range of $200-500 \mu \mathrm{m}$ are described. In addition, numerical models based on the finite element (FE) method 
were constructed to further understand the mechanisms of conduction. The results from these different approaches are presented and discussed.

\section{EXPERIMENTAL METHODS}

\section{A. Experimental Linearity Measurements}

Electrical measurements were made on a number of assemblies to observe the linearity of the contact resistance of the flip-chip assemblies joints. These assemblies were constructed with devices of geometries in the range of 200-500 $\mu \mathrm{m}$ using the specially developed instrumented assembly system. The instrumented assembly system allows for the measurement of process parameters during and after assembly. The details of the assembly system including the flip-chip assembly methods are given in [2]. Two basic types of substrates were used:

1) organic substrates of $\mathrm{Cu}$ clad FR5 laminate with an electroless nickel/gold coating on the $\mathrm{Cu}$;

2) ceramic substrates made of alumina having a thick film AgPd metallization.

The flip chips were assembled onto these substrates with three different adhesives, namely

1) thermosetting adhesive resin with $5 \mu \mathrm{m}$ diameter polymer spheres coated with nickel (5 $\mu \mathrm{m}$ Set-Polycore);

2) thermosetting adhesive resin with $2 \mu \mathrm{m}$ solid nickel spherical filler ( $2 \mu \mathrm{m}$ Set-Nickel);

3) thermoplastic adhesive resin with $10 \mu \mathrm{m}$ polymer spheres coated with gold (10 $\mu \mathrm{m}$ Plastic-Polycore).

The components were $10 \mathrm{~mm}$ square ceramic devices with $500 \mu \mathrm{m}$ pitch array connections, representative of a $\mu \mathrm{BGA}$. In a previous study [3], it was concluded that uniform conductivity and high yield are more readily obtained with the organic substrates than with the thick-film on ceramic substrates owing to the differences in the topology of the pads and the mating bump on the component. It was considered that the flat top of the metallization on the organic substrate makes it easy to retain a significant number of conducting particles required for electrical conduction, and that gold-coated copper PCB pads are more consistent in shape than those on the thickfilm ceramic substrates. For these reasons, efforts were initially geared toward constructing assemblies with the PCB's.

The resistance linearity measurements were aimed at establishing the electrical behavior of both low and high resistance conducting joints, across the various types of ACA's and substrates used in the assemblies. Using the four-point probe method measurements were made by varying the current source and observing the corresponding voltage across the joints. Stationary contact was assumed for the contact surface since there was no application of external pressure during the measurements. The results from these measurements are presented and discussed below.

\section{NUMERICAL METHOD}

\section{A. Electric Contact Theory}

It is of interest to review the mode of contact when two metal conductors are brought into contact by an applied force because of their importance in determining contact resistance.
When such conductors are placed in contact they would make an initial contact at minute spots distributed over the mating surfaces. This is because no surface is truly smooth, flat or planar; actual contact surfaces are characterized by a certain roughness and waviness and, very often, contain a number of individual spots known as asperities. Initial contact of surfaces occurs at these asperities but as force is applied, these contact points will expand into a contact area due to deformation of the contact spots in response to the applied load. The number and size of these individual spots appear to be dependent upon the macroscopic shape and surface finish of the contact members [4], [5]. Electrical contact is made within these small loadbearing asperity areas, the sum of which is regarded as the effective contact area. The effective contact area is therefore an important factor that determines electrical contact resistance.

In flip chip assemblies made using ACA's, electrical conduction is provided through the conducting fillers trapped between the interconnection areas. At the point of contact between the conducting particles and the conducting tracks, the majority of the current will flow through the areas of metalto-metal contact. Thus, current is constricted to flow through these areas and the resistance will therefore be higher than if the current were passing directly through a straight path with no constriction. In addition, heat will be generated at the local contact points due to the current flowing through them. It is also possible that insulating layers such as oxides may be present at contact points [6]. These could give rise to contact resistance variation, nonlinear current-voltage characteristics and temperature instability effects. For the low and stable contact resistance necessary in modern electronic packaging, these effects need investigating.

In order to explore the mechanism of conduction at the electrical joints of the flip chip assembly, including an investigation of the effect of heat generation due to current flow, electrical contact models of the conducting joints in ACA flip chip assembly were constructed using the FE method. In comparison to analytical techniques, the FE technique is suited to the conduction problem because of the complex geometry and boundary conditions while the analytical electrical contact conduction analysis is limited to relatively simple configurations. The electrical contact was modeled as a FE electrical field problem using the analogy between electric and heat conduction.

\section{B. Heat and Electric Conduction Analogy}

Electric current flow in a solid conductor is classically formulated in terms of the electric potential. From Ohm's law, the current density is a vector quantity which is proportional to the gradient of the electric potential, and is given by

$$
I_{i}=\frac{1}{R} \cdot \Delta E
$$

where $R$ is the specific resistance of the solid to current flow. If the current flow is assumed to be mainly dependent on the electric field present, then the governing field equation of the voltage potential problem for the steady-state condition, 
having no charge source, is given by

$$
\frac{1}{R_{x x}} \frac{\partial^{2}}{\partial x^{2}} E+\frac{1}{R_{y y}} \frac{\partial^{2}}{\partial y^{2}} E=0
$$

which, for constant resistivity, reduces to the well-known Laplace equation of the form

$$
\nabla^{2} E=0
$$

where $E$ is the electric potential. In the case of heat conduction, the governing equation (for transient heat conduction) is [7]

$$
K_{x x} \frac{\partial^{2}}{\partial x^{2}} T+K_{y y} \frac{\partial^{2}}{\partial y^{2}} T+\dot{Q}=\rho C \frac{d T}{d t}
$$

where $T$ is the temperature, $\rho$ is the mass density, $C$ is the specific heat capacity, $K$ is the thermal conductivity and $Q$ is the joule heat generation term. For steady-state problem there is no dependency on time and the material is unable to store energy so that the above equation is simplified to

$$
\nabla^{2} T=0 \text {. }
$$

The foregoing equations show that the steady-state electric conduction in a homogenous material of constant resistivity and no internal source of potential (3), is analogous to the steady-state heat conduction (5) in a body of similar geometrical shape. It follows therefore that the dimensionless potential in the electrical conduction system is analogous to the dimensionless temperature in a thermal system. This analogy was exploited in modeling the electrical potential field problem of electrical contact in ACA flip-chip assemblies. The axisymmetric heat conductivity model was used to simulate electrical conductivity in similarly shaped geometry configuration, with imposed electrical voltage simulated by imposed surface temperature.

\section{Finite Element Formulation}

The governing equation of the voltage potential for a steadystate condition, (2) can be changed into the FE form by using the variational formulations. In the variational method the partial differential equations of the field problem are formulated in terms of a variational expression known as the energy functional [8]. In most energy applications, this expression can be identified with stored energy in the system. In general, however, this element equation can be described as

$$
\{F\}=[K]\{E\}
$$

where $[K]$ is the stiffness matrix, $\{F\}$ the force vector and $\{E\}$ is the potential.

\section{Model Geometry and Finite Element Mesh}

A solid sphere representing a typical conductive particle trapped between appropriate substrate and component conductors is shown in Fig. 2. In the present analyzes, a single particle in contact with component metallization and substrate was considered and the effect of multi micro-contacts was neglected. It is difficult to consider the actual rough surface exactly because of its complexity. For this reason, the surface of the contact interface was modeled as an axisymmetric

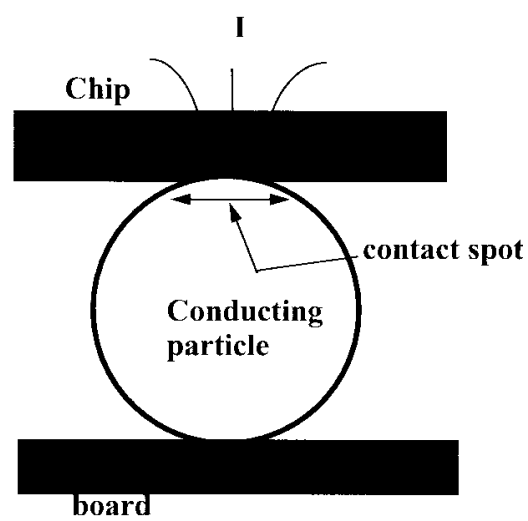

Fig. 2. Schematic representation of a single contact particle showing the contact spot.

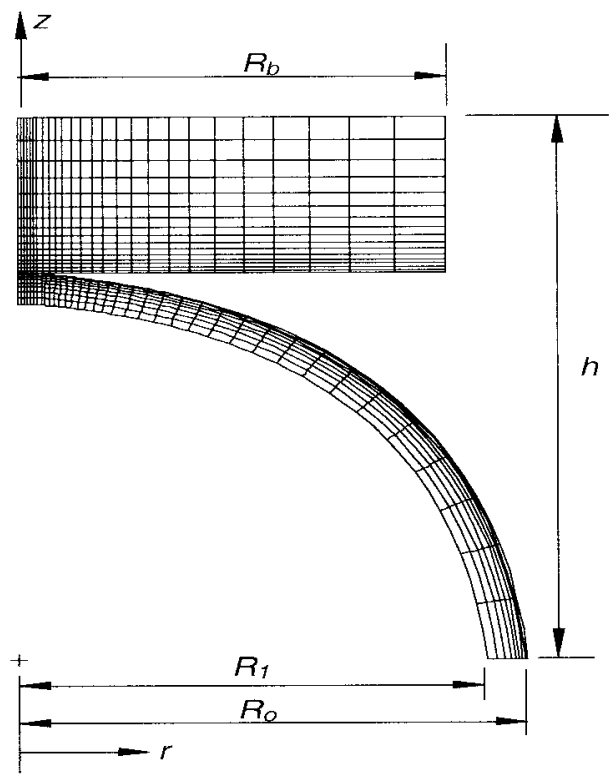

Fig. 3. FE mesh of the metal-coated particle.

surface with the sum total of the individual spots taken as the effective contact area. Taking advantage of symmetry, half of the geometry was modeled using the 8-node axisymmetric, isoparametric elements. Two basic models were developed for the two types of ACA filler particles considered:

1) one for the solid nickel particles;

2) the other for the metal-coated spherical particles.

The contact bodies were meshed with axisymmetric element of the rectangular cross-section, and the boundary conditions of the analyses domain were selected as follows (see Figs. 3 and 4).

1) For the solid sphere

$$
\begin{aligned}
& E=0: \quad 0<r<R_{a}, \quad z=0 \\
& E=V: \quad 0<r<R_{b}, \quad z=h
\end{aligned}
$$

2) For the coated sphere

$$
\begin{aligned}
& E=0: \quad R_{1}<r<R_{0}, \quad z=0 \\
& E=V: \quad 0<r<R_{b}, \quad z=h
\end{aligned}
$$




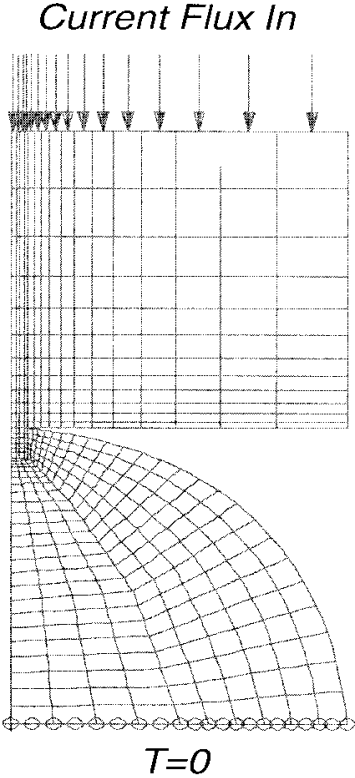

Fig. 4. FE boundary conditions for the solid spherical conducting particle.

where $R_{a}$ is the radius of the solid sphere, $R_{b}$ is the length of the conducting track, $h$ is the height of the analysis domain, while $R_{0}$ and $R_{1}$, respectively are the inner and outer radius of the coated sphere. The total number of elements used in the analyses depended on the size of the contact radius. In all the models, the FE mesh was graded so that element sizes were smallest in the region of the contact zone, i.e., where the highest current densities were expected to occur. Different contact sizes were accommodated by modifying the contact radius and applying the current to more or less nodes on the surface. Because of axisymmetry, the inner boundary was assumed to be insulated, likewise, the portion of the contact's face where current is not applied was also assumed to be insulated. The backside was not insulated and was taken to be at zero potential as shown in Fig. 4. The problem was solved for different contact radii.

\section{RESUlTS AND DisCUSSION}

\section{A. Experimental Linearity Measurements}

A typical representative plot of the resistance-voltage (RV) characteristics of the assemblies is shown in Fig. 5. The measurements were made to reflect the joints of the various types of ACA's and substrates used in constructing the assemblies. The results from these measurements show four observable characteristics.

1) Ohmic (linear) behavior-constant resistance for rising current.

2) Non-Ohmic behavior showing a rising resistance with increasing voltage.

3) Decreasing resistance as current rises.

4) Rising resistance at low currents followed by a drop in resistance.

The linearity measurements show that in most cases, the low resistance $(<1 \Omega)$ joints exhibit Ohmic behavior, i.e., constant

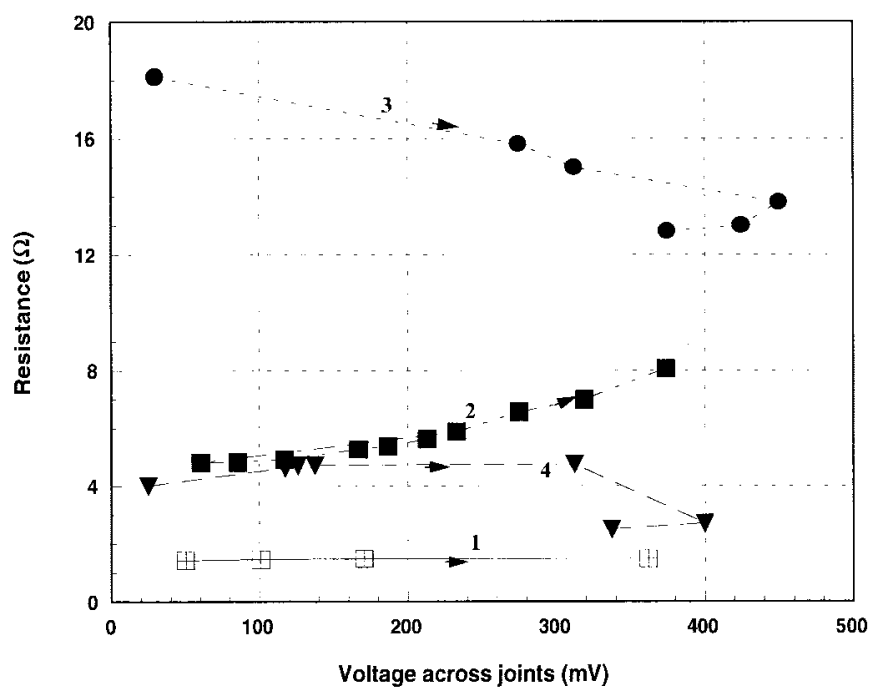

Fig. 5. Typical resistance-voltage characteristics of $\mu \mathrm{BGA}$ assemblies joints.

resistance over the current carrying capacity of the joints. The Ohmic behavior at the low resistance joints clearly indicates that the nature of the contact was metallic.

In some of the high resistance joints, linearity measurements show that the resistance increases with rising current. In the other high resistance joints, there was some indication of initial ohmic behavior followed by some nonlinear behavior due to either resistive heating or rupture of insulating film at contact make. For example, joints of 3.8 and $3.9 \Omega$ initial resistances show an initial rising RV-characteristics followed by a drop in resistance. This drop could be associated with an increase in the contact area. With increasing current passing through the contact, the temperature in the contact surface would rise by a corresponding amount. When the softening temperature of the contact is reached, the contact area would increase, and the contact resistance would decrease, causing a drop in the RV-characteristics. Holm [4] refers to this decrease as the "softening drop." It is also possible that the drop in resistance could be as a result of a breakdown of some insulating film at the contact surfaces which increases the effective contact surface area available for conduction. A non-Ohmic behavior having a seemingly exponential drop in resistance was also observed in some of the high resistance joints. The nonlinear behavior observed in most of the high resistance joints gives a strong indication of a presence of an insulating film on the contact surfaces of these joints.

Similar behavior has also been observed in assemblies constructed with $5 \mathrm{~mm}^{2}$ flip-chip devices of $220 \mu \mathrm{m}$ pitch daisy chained connections. This mechanism is explored further through the computational models.

1) Reversible Characteristics: Measurements were also made to observe any reversible RV-characteristics that would occur in some of the joints. It should be noted that in order to obtain reversible characteristics the current must first be ramped-down followed by a ramped-up measurement. A way of achieving this would be to ensure that the temperature due to current flow was far below the melting point of the contact surface materials, and to use fairly short current transients. 


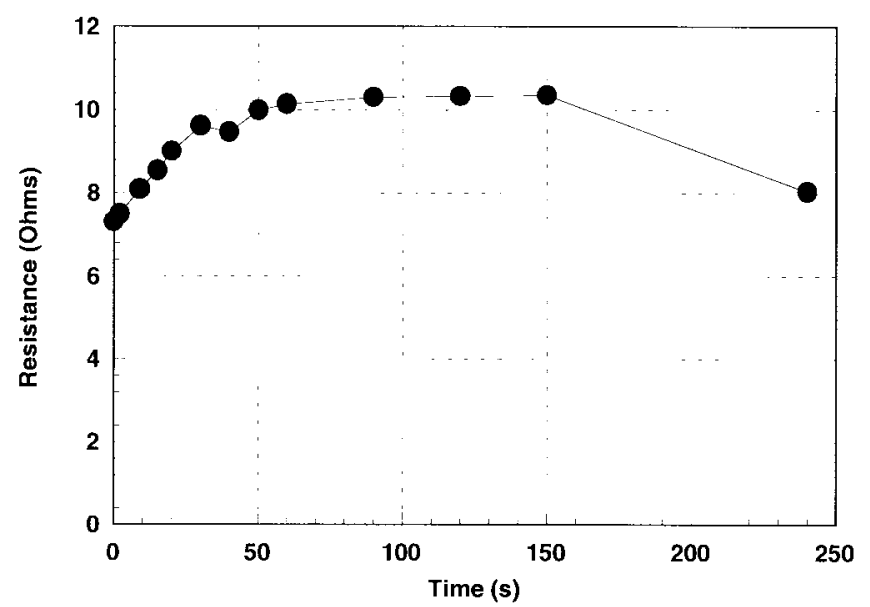

Fig. 6. Variation of resistance with time at constant voltage.

Unfortunately, it was not possible to establish the melting temperature (and melting voltage) of the contact surface materials prior to measurements. There was also some problem in determining the current carrying capacity of most of the joints prior to measurements, as a result some of the joints suffered from open circuiting due to the passing of excessive current through the joints. However, some of the measured $\mathrm{RV}$-characteristics showed some reversible behavior.

2) Temperature Effects: It is of importance to understand the effects temperature variation would have on joint resistance and how temperature is distributed in the constriction of the contact spots at various stages of heating due to current flow. Joint resistance measurements made at temperature variations of $4-55^{\circ} \mathrm{C}$ did not show any significant change and no change to open circuit was observed. However, previous temperature cycling reliability experiments [9] on assemblies of similar adhesive types have shown that temperature cycling does indeed affect the joint resistance. Nevertheless the plot in Fig. 6 shows the variation of joint resistance with time at constant voltage. The graph exhibits an initial increase in joint resistance for a period of $60 \mathrm{~s}$, remains constant for another $100 \mathrm{~s}$ and then dropped on removal of the current source. The phenomena behind this resistance variation is not yet certain, but is probably due to heating effects from the initial current flow. The result does however suggest the need to apply short current transients when measuring joint resistance behavior so as to avoid heating effects. However, more investigation is needed to enable definite conclusion to be made.

\section{B. Numerical Results}

In the numerical study, the simulation results are given in terms of the electric field response occurring at the contact zone. The numerical models were verified so as to confirm the validity of the proposed technique. Fig. 7 shows the comparison of the FE computed contact resistances with those from the well-known Holm model of conduction through a solid sphere [4]. Excellent agreement is obtained between the two, thus, giving confidence in the FE modeling technique.

The voltage potential and current density distributions at the contact zone of the solid spherical ACA particle and for

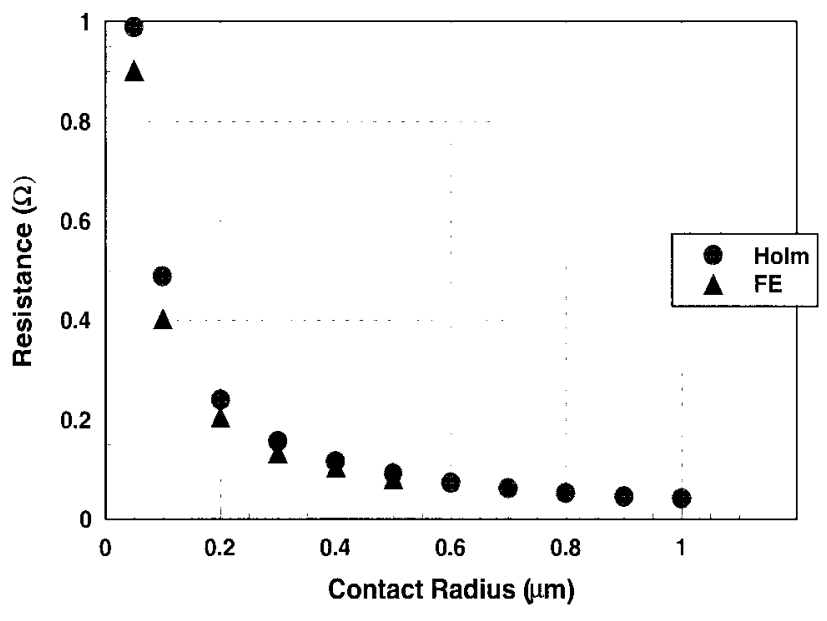

Fig. 7. Comparison of results from the FE model with those from Holm's model of conduction.

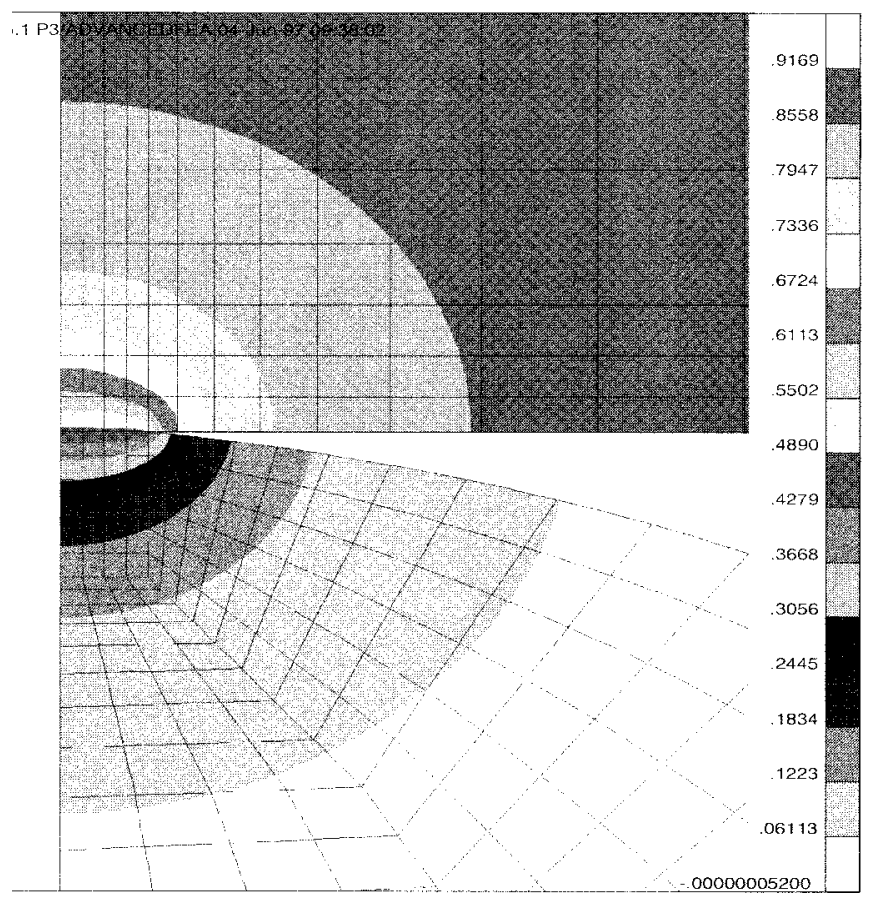

Fig. 8. Voltage potential distribution at the contact zone of the solid sphere $(V)$.

the contact conditions, $a=0.05 \mu \mathrm{m}$ are plotted in Figs. 8 and 9 , respectively. For a current flow of $1.0 \mathrm{~A}$, the contact resistance for the contact conditions, $a=0.05 \mu \mathrm{m}$ is $0.92 \Omega$. This is against $0.46 \Omega$, for $a=0.10 \mu \mathrm{m}$ indicating that the higher contact area gives a lower contact resistance. This is in an agreement with previous studies which show that bonding pressure plays an important role in the electrical properties of ACA interconnections [10]. At the contact interface, nearly parallel isopotential lines were drawn, an indication that the distribution of the current flow is nearly uniform in that zone.

A careful study of the current density distribution shows that there is a high current density at the contact interface, as would be expected. If the potential distribution is obtained, the joule 


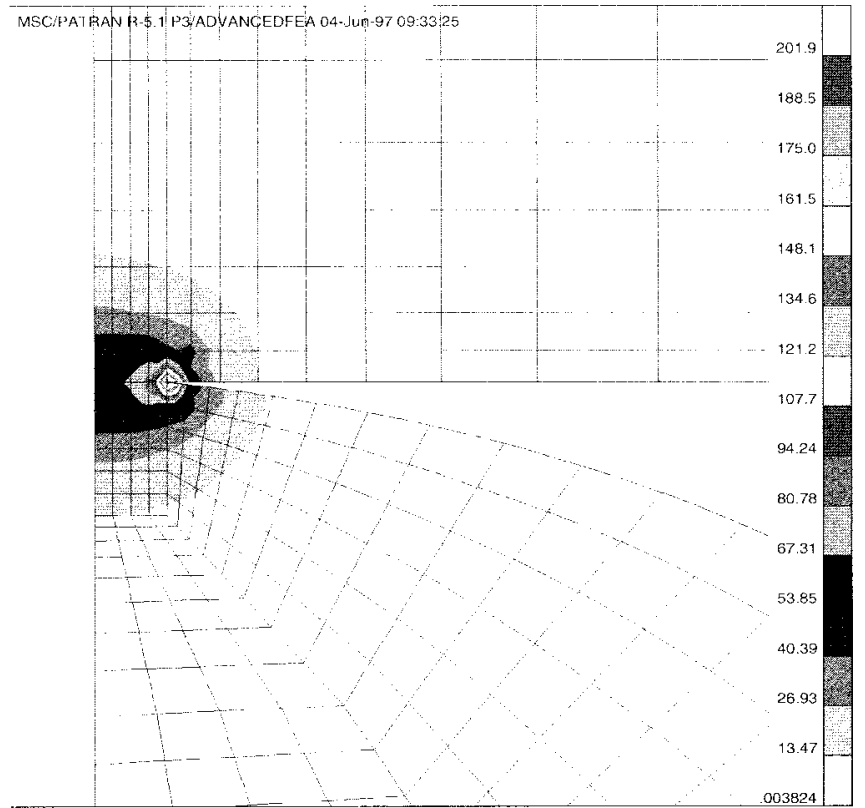

Fig. 9. Current density distribution for the solid sphere $\left(\mathrm{A} / \mu \mathrm{m}^{2}\right)$.

heat generation per unit volume, $Q$, can be calculated by using

$$
Q=J^{2} \rho
$$

where $J$ is the current density, and $\rho$ is the electrical resistivity. Fig. 10 shows a plot of the contours of joule heat generation for the solid sphere and contact radius of $0.05 \mu \mathrm{m}$. A comparison between the different contact radii shows that the amount of heat generation for the $0.05 \mu \mathrm{m}$ contact is higher than that from the $0.10 \mu \mathrm{m}$ by more than a factor of ten. The results indicate that increasing the contact radius by a factor of two results in a decrease in the amount of heat generation per unit volume by a factor of more than ten. This is in contrast with the contact resistance values which shows a relationship of a factor of two in going from a contact radius of $0.10-0.05 \mu \mathrm{m}$, indicating that the effect of contact radius variation is more pronounced with respect to heat generation than with contact resistance.

The maximum temperature increment due to the local heating effects can be estimated from the constriction voltage by means of the following expression [4]:

$$
V^{2}=8 \int_{T_{1}}^{T_{2}} \rho K d T
$$

where $K$ is the thermal conductivity. For a long constriction with the total voltage, $V,(8)$ reduces to

$$
\Delta T=\frac{1}{2} V \sqrt{\frac{1}{L}}
$$

where $L=\rho K$ is the Lorenz constant. Using the above relations, the computed temperature rise for the contact conditions of $a=0.05 \mu \mathrm{m}$ and $a=0.10 \mu \mathrm{m}$, are 195 and

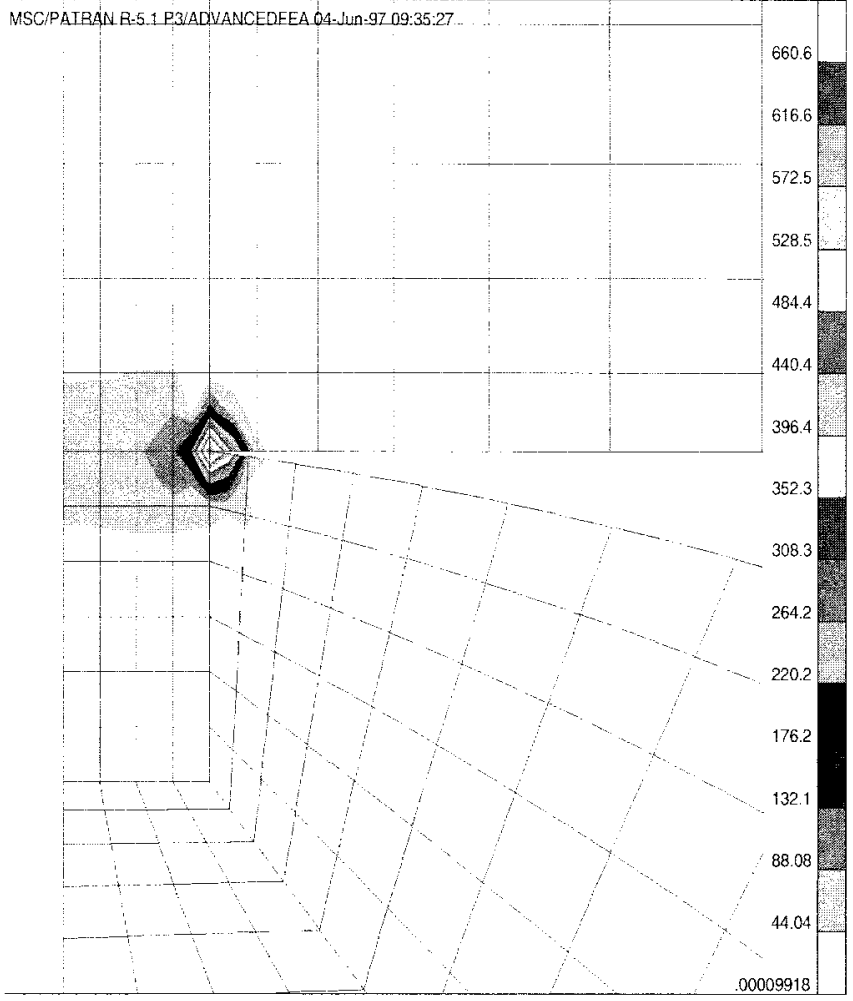

Fig. 10. Joule heat distribution for the solid sphere $\left(\mathrm{W} / \mu \mathrm{m}^{3}\right)$.

$100{ }^{\circ} \mathrm{C}$, respectively. When the contact surfaces are heated by a current and if both contact members are of the same material, the highest temperature would be expected to be localized within the contact surface and distant points would correspondingly be at lower temperatures. Plots of the current density distribution and those of heat generation show that this is indeed the case. It is expected that melting will start to occur at the point where the current density is high. It is however expected that with melting the area of contact would increase resulting in a corresponding current density decrease. It would then take more current to initiate melting when the contact area is larger.

Different FE models were built to determine the contact areas that would give the high resistance joints observed in the experimental trials. Table I summarizes the FE predicted contact resistances and the resulting maximum temperature rise due to joule heating, for the $2 \mu \mathrm{m}$ Set-Nickel particle. It shows that the contact areas required for high resistance values are extremely small and that the temperature rise for these small areas are high. For example, Table I shows that to obtain a $1 \Omega$ resistance requires a contact diameter of about $0.1 \mu \mathrm{m}$. This is very small indeed, and would result to resistive heating of the contact surfaces due to high current densities occurring at the small contact area.

In order to compare the advantage of using one type of ACA particle over another, the model was extended to those of an insulating sphere coated with nickel representing the $5 \mu \mathrm{m}$ Set-Polycore (polymer cored Ni) particle. Plots of the contours of the potential distribution for the $0.10 \mu \mathrm{m}$ contact radius are shown in Fig. 11, while those for the current 
TABLE I

PREDicted CONTACT RESISTANCE AND TEMPERATURE RISE FOR THE $2 \mu \mathrm{m}$ SET-NicKel

\begin{tabular}{c|c|c}
\hline $\begin{array}{c}\text { Contact } \\
\text { radius } a(\mu \mathrm{m})\end{array}$ & $\begin{array}{c}\text { Resistance } \\
(\Omega)\end{array}$ & $\begin{array}{c}\text { Temperature } \\
\text { rise }\left({ }^{\circ} \mathbf{C}\right)\end{array}$ \\
\hline \hline 0.05 & 0.917 & 194.61 \\
\hline 0.10 & 0.464 & 98.47 \\
\hline 0.20 & 0.234 & 49.66 \\
\hline 0.30 & 0.155 & 32.89 \\
\hline 0.40 & 0.112 & 23.76 \\
\hline 0.50 & 0.097 & 20.59 \\
\hline 0.60 & 0.072 & 15.28 \\
\hline
\end{tabular}

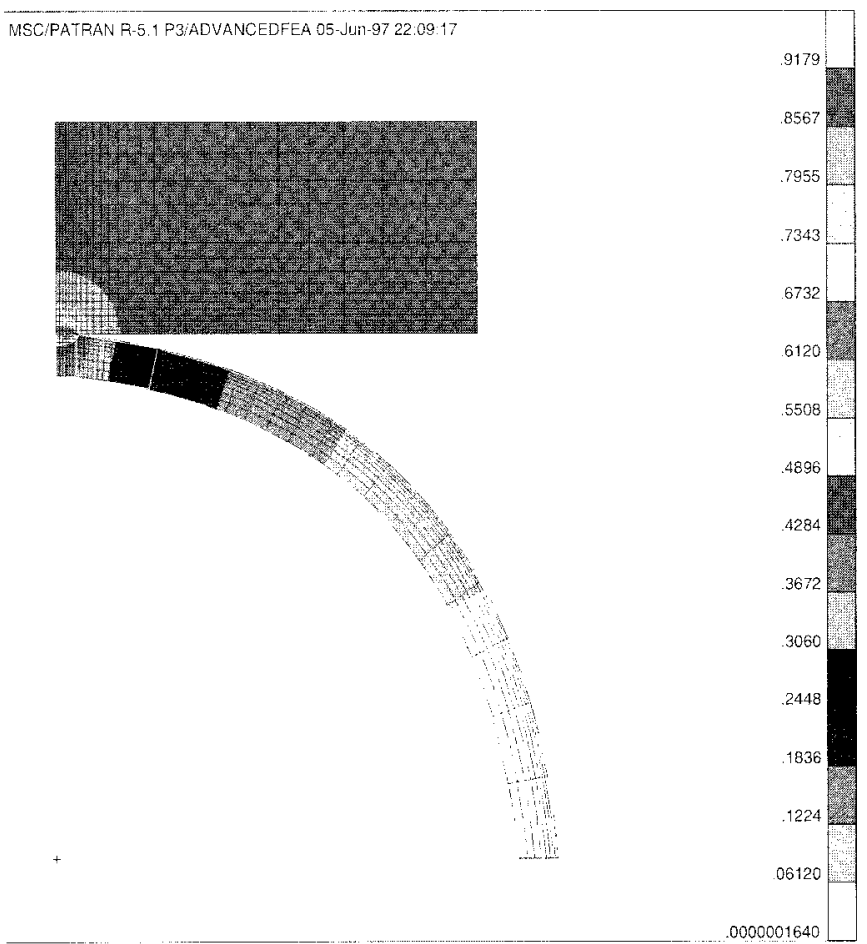

Fig. 11. Voltage Potential distribution for the metal-coated insulating sphere $(V)$.

density and joule heat generation distributions are shown in Figs. 12 and 13, respectively. The maximum temperature rise due to local heating computed for this case is $195{ }^{\circ} \mathrm{C}$. The FE predicted contact resistances and the computed maximum temperature rise due to joule heating, are summarized in Table II. These results also show that the resistances required for high resistance values are equally small for the metalcoated polymer sphere.

Comparing the $2 \mu \mathrm{m}$ Set-Nickel spherical particle with the $5 \mu \mathrm{m}$ Set-Polymer cored particle with the same degree of contact, it is clear that the $2 \mu \mathrm{m}$ Set-Nickel sphere has a lower contact resistance and temperature rise. The maximum joule heat generated per unit volume at the contact spot is also higher in the $5 \mu \mathrm{m}$ Set-Polymer cored particle. It can therefore be concluded that in terms of the FE predicted field variables, the performance of the $2 \mu \mathrm{m}$ Set-Nickel sphere is better than the

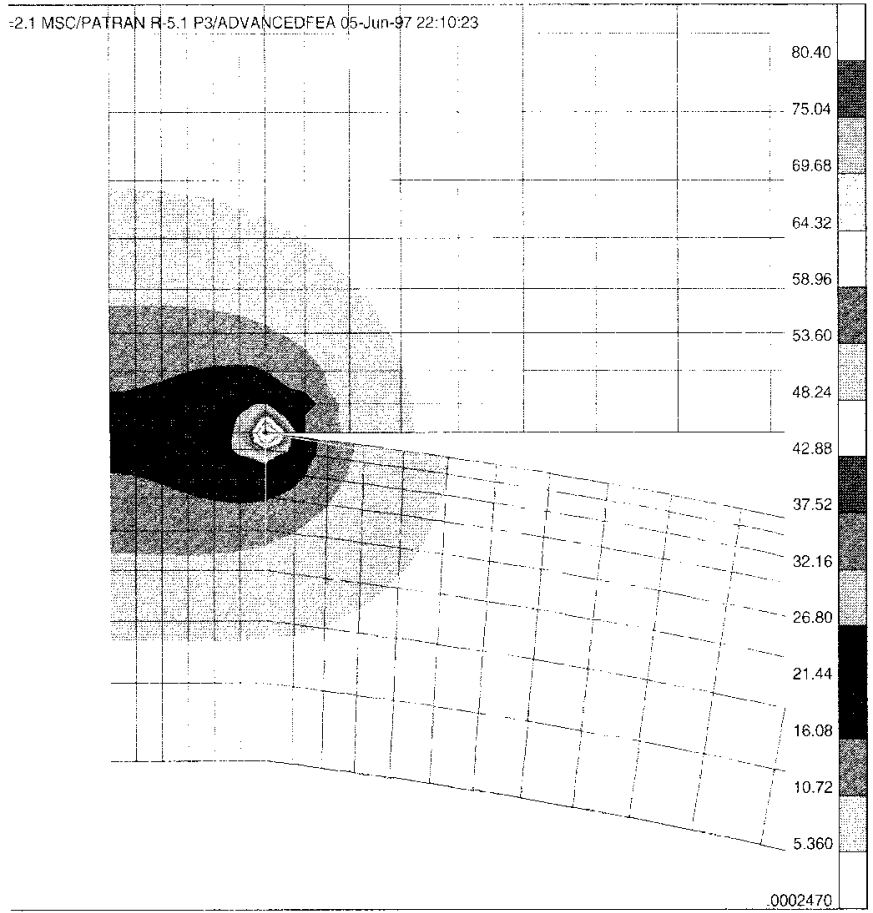

Fig. 12. Current density distribution for the metal-coated insulating sphere $\left(\mathrm{A} / \mu \mathrm{m}^{2}\right)$.

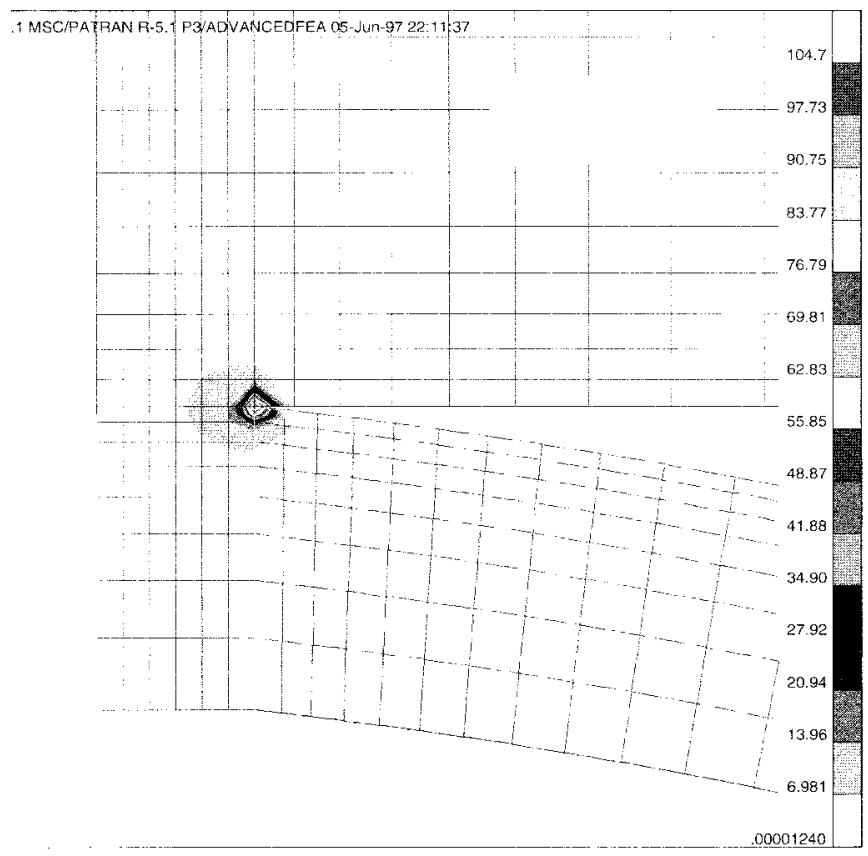

Fig. 13. Joule heat distribution for the metal-coated insulating sphere $\left(\mathrm{W} / \mu \mathrm{m}^{3}\right)$.

$5 \mu \mathrm{m}$ Set-Polycore sphere. However, the $5 \mu \mathrm{m}$ Set-Polycore sphere offers some other advantage such as better ability to absorb nonplanarity in the assemblies.

In general the models have shown that the contact areas required for high resistances are extremely small for both the solid nickel and the polymer cored particles. The likelihood of obtaining high resistance through these small areas of contact without burning it off due to the high temperature rise and high heat generation is small, giving a strong indication that thin 
TABLE II

PREDicted CONTACT ResistanCE AND Temperature Rise For the $5 \mu \mathrm{m}$ Set-Polycore

\begin{tabular}{c|c|c}
\hline $\begin{array}{c}\text { Contact } \\
\text { radius } a(\mu \mathrm{m})\end{array}$ & $\begin{array}{c}\text { Resistance } \\
(\Omega)\end{array}$ & $\begin{array}{c}\text { Temperature } \\
\text { rise }\left({ }^{\circ} \mathbf{C}\right)\end{array}$ \\
\hline 0.05 & 1.829 & 388.15 \\
\hline 0.10 & 0.918 & 194.82 \\
\hline 0.20 & 0.407 & 86.37 \\
\hline 0.30 & 0.317 & 67.27 \\
\hline 0.40 & 0.211 & 44.78 \\
\hline
\end{tabular}

films are present at the contact surfaces of the joints exhibiting high resistivity.

\section{CONCLUSION}

We have reported on the experimental and numerical activities aimed at understanding the issues involved in conductivity of the ACA flip chip technology. Experimental measurements that investigated the linearity of the contact resistance of the conducting joints have shown that most of the joints with low resistance are Ohmic. Some of the high resistance joints show some resistive heating while others indicated an initial Ohmic behavior followed by some exponential drop in their contact resistance. FE numerical models have predicted behavior in metallic joints which indicate that some thin layers of insulating films must be present at the contact surfaces of the joints showing high resistance.

The FE study has further demonstrated the feasibility of solving complex electrical conductivity problems related to contacts. It has also served to underline the need for more accurate information about the mechanism of contact. One improvement to the modeling of the electric contact problem could be to fully couple the electrical contact problem to the heat conduction problem. The joule heat generation rate distribution of the conducting body could be applied in determining the thermal response of the contact joints. A further improvement would be in the modeling of problems where thin films are present at the surface of the contact points. This would however require additional information about the nature of the films.

\section{REFERENCES}

[1] A. O. Ogunjimi et al., "A review of the impact of conductive adhesive technology on interconnection," J. Electron. Manufact., vol. 2, pp. $109-118,1992$.

[2] _ "Assembly of planar array components using anisotropic conducting adhesives-A benchmark study: Part I-Experiment," IEEE Trans. Comp., Packag., Manufact. Technol., vol. 19, pp. 257-263, Oct. 1996.

[3] S. H. Mannan et al., "Assembly of planar array components using anisotropic conducting adhesives-A benchmark study: Part II-Theory," IEEE Trans. Comp., Packag., Manufact. Technol., vol. 19, pp. 264-269, Oct. 1997.

[4] R. Holm, Electric Contacts, Theory and Application, 4th ed. Berlin, Germany: Springer-Verlag, 1967.

[5] K. L. Johnson, Contact Mechanics. Cambridge, U.K.: Cambridge Univ. Press, 1987.

[6] J. Liu, et al., "Surface characteristics, reliability and failure mechanisms of tin, copper and gold metallizations," in Proc. Adhesives Electron. 96, Stockholm, Sweden, 1996, pp. 141-153.

[7] A. J. Chapman, Heat Transfer, 3rd ed. New York: Macmillian, 1974.
[8] M. V. K. Chari, Ed., Finite elements in Electrical and Magnetic Field Problems. New York: Wiley, 1980.

[9] S. H. Mannan et al., "Electrical contact reliability in anisotropic conducting adhesives connections for flip chip technology," in Proc. InterPack '97, Hawaii, June 1997, pp. 167-176.

[10] M. J. Yim et al., "A study on the electrical conduction mechanism of anisotropically conductive film (ACF) for LCD packaging applications," in Proc. InterPack '97, Hawaii, June 1997, pp. 65-72.

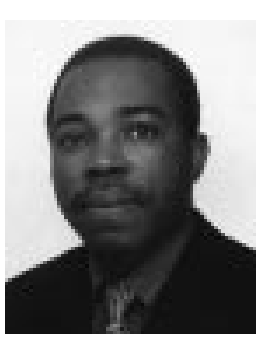

Chuks N. Oguibe received the B.Eng. degree in mechanical engineering from the Federal University of Technology, Owerri, Nigeria, the M.Sc. degree in mechanical computer-aided engineering from the University of Strathclyde, U.K., and the Ph.D. degree in computational mechanics from Leeds Metropolitan University, U.K.

$\mathrm{He}$ is a Postdoctoral Research Associate in the Department of Manufacturing Engineering, Loughborough University, U.K., where he is working in the area of electronics manufacturing process modeling and reliability physics. His research interest focuses on computational modeling of electronics manufacturing processes, including reliability physics.

Dr. Oguibe is an Associate Member of ASME.

Samjid H. Mannan received the B.A. degree in physics from Oxford University, Oxford, U.K., in 1988 and the Ph.D. degree in theoretical elementary particle physics from Southampton University, U.K., in 1991.

After working as a Research Assistant at Salford University and Loughborough University in the area of electronics interconnection (solder paste and ACA's), he is currently working in the area of electronics manufacturing as a Research Fellow at Loughborough University, U.K. He is the author of over 20 scientific papers.

Dr. Mannan is a Chartered Physicist and a member of the Institute of Physics.

David C. Whalley received the B.Sc. and M.Phil. degrees from Loughborough University, U.K.

Since then, he has been involved in research into electronics interconnection reliability, the use of engineering analysis techniques both in electronic product design, and in process simulation, and on new electronics interconnection technologies such as conducting adhesives. He has worked as an Engineer both at Loughborough University, and at Lucas Industries' Advanced Engineering Centre, Solihul, Birmingham, U.K., before being appointed in 1990 as Lecturer in Processes for Electronic Manufacture, Department of Manufacturing Engineering, Loughborough University. $\mathrm{He}$ is the author of over 60 papers in the areas of electronics manufacturing processes, interconnection technology, and electronic thermal design.

David J. Williams received the degree in mechanical engineering (with first class honors) from the Institute of Science and Technology, University of Manchester, Manchester, U.K., and the Ph.D degree from Cambridge University, Cambridge, U.K.

$\mathrm{He}$ has been Professor of Manufacturing Processes, Loughborough University, since January 1989. He is Director of the PRIME Faraday partnership. Four Faraday partnership have been created in the U.K. to improve bridges academia and industry. The PRIME Faraday partnership addresses issues associated with the design and manufacture of complex, hybrid systems products where electronic and mechanical subsystems are interdependent. His present research focuses on the resolution of problems within electronics manufacturing. This work includes work at the process engineering science level, understanding of the interaction between process understanding and design for manufacture (encompassing take back), and business globalization. Before taking up his present post, he was a Lecturer in Manufacturing Engineering and Design, Engineering Department, Cambridge University, U.K., and worked in industry for Metal Box and GKN. 